\title{
Nonsustained Supraventricular Tachycardia by ECG Finding
}

National Cancer Institute

\section{Source}

National Cancer Institute. Nonsustained Supraventricular Tachycardia by ECG Finding. NCI Thesaurus. Code C120614.

An electrocardiographic tracing demonstrating supraventricular tachycardia of less than thirty seconds duration. Different thresholds, for rate and/or duration, may apply to different patient populations. 